\title{
SOME EXAMPLES OF DISCRETE GROUP ACTIONS ON ASPHERICAL MANIFOLDS
}

\author{
M. W. DAVIS AND I. J. LEARY
}

\begin{abstract}
We construct two classes of examples of a virtually torsionfree group $G$ acting properly and cocompactly on a contractible manifold $X$. In the first class of examples the universal space for proper actions, $\underline{E} G$, has no model with finitely many orbits of cells. The reason is that the centralizers of certain finite subgroups of $G$ will not have finitetype classifying spaces. In the second class of examples $X$ is a $C A T(0)$ manifold upon which $G$ acts by isometries. It follows that $X$ is a model for $\underline{E} G$. In these examples the fixed point sets of certain finite subgroups of $G$ are not manifolds and the centralizers of these subgroups are not virtual Poincaré duality groups.
\end{abstract}

\section{INTRODUCTION}

A discrete group $G$ is type $F$ if its classifying space $B G$ (also called its "Eilenberg-Mac Lane space") has the homotopy type of a finite complex. (There is a weaker, algebraic version of this condition known as "type FP"; the definition can be found in [5].) A group of type F (or FP) is necessarily torsion-free. A virtually torsion-free group $G$ is type $V F$ (resp. type VFP) if it contains a finite index subgroup of type $\mathrm{F}$ (resp. of type FP).

For any discrete group $G$, there is a CW-complex $\underline{E} G$ on which $G$ acts cellularly and properly (i.e., with finite isotropy subgroups) so that for each subgroup $H$ of $G$, the fixed set $(\underline{E} G)^{H}$ is contractible whenever $H$ is finite (and is empty when $H$ is infinite). $\underline{E} G$ is called the universal space for proper $G$-actions. It is unique up to $G$-homotopy equivalence. (See [19].) We say that $G$ is type $\mathcal{V} F$ if there is an $E G$ so that the quotient space $E G / G$ is compact. A group $G$ of type $\mathcal{V} F$ is not necessarily virtually torsion-free (see, for example, [2, p. 493]), but any virtually torsion-free group of type $\mathcal{V} F$ is of type $\mathrm{VF}$. There is also a similar, but weaker, algebraic notion of a group being of "type $\mathcal{V} F P$ ", which will be defined in an appendix to this article.

Date: January 15, 2004.

2000 Mathematics Subject Classification. Primary: 57S30; Secondary: 20F36, 20F55, 57M07.

Key words and phrases. finiteness conditions, Poincaré duality group.

The first author was partially supported by NSF grant DMS-0104026. The second author acknowledges support from EPSRC (via grant GR/R07813), and from the Ohio State Mathematical Research Institute. 
In many of the most well-known examples of groups of type VF or of type $\mathcal{V} F, \underline{E} G$ is a contractible manifold. For example, suppose that $X$ is a simply connected, complete Riemannian manifold of nonpositive sectional curvature (e.g., a symmetric space) and that $G$ is a discrete group of isometries of $X$. For each finite subgroup $H$, the nonpositive curvature condition forces the fixed set $X^{H}$ to be nonempty and convex and hence, to be contractible. The same argument works even when the metric is singular, i.e., when $X$ is a "CAT(0) space". (See [4] for the definitions and basic facts concerning $C A T(0)$ spaces.) Thus, if $G$ is a discrete group of isometries of a $C A T(0)$ $X$, then $X$ is a model for $\underline{E} G$. In particular, if the quotient space $X / G$ is compact, then $G$ is type $\mathcal{V} F$. We note that, in the Riemannian case, since the $G$-action is smooth, each fixed set $X^{H}$ is a smooth submanifold of $X$.

In [13] the second author and B. Nucinkis produced the first examples of groups of type $\mathrm{VF}$ which were not of type $\mathcal{V} F$. How does one tell that a given group $G$ is not of type $\mathcal{V} F$ ? For any finite subgroup $H$ of $G$, let $V_{H}=$ $N_{G}(H) / H$, where $N_{G}(H)$ stands the normalizer of $H$ in $G$. Then $(E G)^{H}$ is

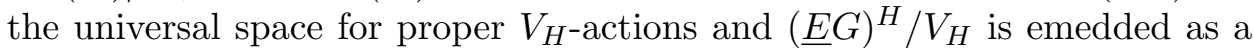
closed subspace of $E G / G$. It follows from this, first of all, that if $G$ is type $\mathcal{V} F$, then (since $\underline{E} G / G$ is compact) there are only finitely many conjugacy classes of finite subgroups in $G$. Secondly, if $G$ is type $\mathcal{V} F$, then so is $V_{H}$. In particular, if $V_{H}$ contains a torsion-free subgroup of finite index which is not type $\mathrm{F}$, then $G$ cannot be type $\mathcal{V} F$. In [13] various examples of $G$ of type VF are constructed which violate both these conditions. The method of [13] is to combine the construction of Bestvina-Brady [1] with earlier examples (e.g., of Oliver [17]) of exotic actions of finite groups on contractible finite complexes.

In [9] the first author showed how the reflection group trick could be used to promote the Bestvina-Brady examples to examples of Poincaré duality groups which cannot be finitely presented. In our first class of examples we show how the same trick can be used to promote the examples of [13] to examples where the virtually torsion-free group $G$ acts cocompactly on a contractible manifold, thereby proving the following theorem.

Theorem 1. There are examples of virtually torsion-free groups $G$ such that $G$ acts properly and cocompactly on a contractible manifold and such that $G$ is not of type $\mathcal{V} F$.

A discrete group $G$ is a $P D^{n}$-group (or a "Poincaŕe duality group") if it is type $\mathrm{FP}$ and if

$$
H^{i}(G ; \mathbb{Z} G)= \begin{cases}\mathbb{Z}, & \text { if } i=n, \\ 0, & \text { otherwise. }\end{cases}
$$

It follows that a group of type $\mathrm{F}$ is a $P D^{n}$-group if and only if its classifying space is a Poincaré complex. A virtually torsion-free group $G$ is a $V P D^{n}$ group (a "virtual Poincaré duality group") if it contains a subgroup of finite index which is a $P D^{n}$-group. 
Suppose that $G$ admits a $\underline{E} G$ which is a manifold with cocompact $G$ action (this of course implies that $G$ is of type $\mathcal{V} F$ ). If for each finite subgroup $H,(\underline{E} G)^{H}$ is also a contractible manifold, then the cohomology with compact supports of $(\underline{E} G)^{H}$ is concentrated in the top dimension (and is isomorphic to $\mathbb{Z}$ in that dimension). This dimension is, in fact, $c d_{\mathbb{Q}}\left(V_{H}\right)$ (where $c d_{\mathbb{Q}}(-)$ stands for "cohomological dimension over $\mathbb{Q}$ "). We also note that the cohomology with compact supports of $(\underline{E} G)^{H}$ is isomorphic to $H^{*}\left(V_{H} ; \mathbb{Z} V_{H}\right)$ (see Exercise 4, p. 209 in [5]). This motivates the following definition.

Definition. A group $G$ is a $\mathcal{V} P D$-group if it is type $\mathcal{V} F P$ and if for each finite subgroup $H$

$$
H^{i}\left(V_{H} ; \mathbb{Z} V_{H}\right)= \begin{cases}\mathbb{Z}, & \text { if } i=c d_{\mathbb{Q}}\left(V_{H}\right), \\ 0, & \text { otherwise. }\end{cases}
$$

The above definition relies on the definition of type $\mathcal{V} F P$, which we include in an appendix, as it is rather technical. We shall apply the above definition only in the case when $G$ is type $\mathcal{V} F$, which implies that $G$ is type $\mathcal{V} F P$. Hence the reader who is not interested in algebra will be able to read the rest of this article without reading the appendix.

In our second class of examples $G$ will be a virtually torsion-free group acting cocompactly on a $C A T(0)$ manifold $X$ (so $G$ is type $\mathcal{V} F$ ) yet $G$ is not a $\mathcal{V P D}$-group. The point is that for certain finite subgroups $H$, the fixed set $X^{H}$ will not be a submanifold and will not satisfy Poincaré duality. In these examples the group $G$ will be the semidirect product of a right-angled Coxeter group and a finite group. So, we will have proved the following.

Theorem 2. There are examples of virtually torsion-free groups $G$ such that $G$ is a discrete, cocompact group of isometries of a CAT(0) manifold and such that $G$ is not a $\mathcal{V} P D$-group.

\section{The EQUiVARIANT REFLECTION GROUP TRICK}

A simplicial complex $L$ is a flag complex if any finite set of vertices which are pairwise connected by edges spans a simplex of $L$.

Suppose we are given as data:

- a space $M$ and a subspace $N$ such that $N$ is triangulated as a finite dimensional flag complex,

- the action of a discrete group $G$ on $M$ so that $G$ stabilizes $N$ and so that $G$ acts on $N$ by simplicial automorphisms.

To this data we will associate a right-angled Coxeter group $W$, a space $\mathcal{U}(M, N, G)$ and an action of the semidirect product $W \rtimes G$ on $\mathcal{U}(M, N, G)$. (In practice, in this paper, $M$ will always be a manifold with boundary and $N=\partial M$.)

Let I denote the vertex set of the triangulation of $N$. W is defined by a presentation as follows. There is one generator $s_{i}$ for each $i \in I$. The 
relations are given by $s_{i}^{2}=1$, for $i \in I$, and $\left(s_{i} s_{j}\right)^{2}=1$ whenever $\{i, j\}$ spans an edge of $N$. The $G$-action on $I$ induces an action of $G$ on $W$ by automorphisms. Hence, we can form the semidirect product $W \rtimes G$.

For each vertex $i$, let $N_{i}$ denote the closed star of $i$ in the barycentric subdivision of $N$. For each $x \in N$, let $\sigma(x)=\left\{i \in I \mid x \in N_{i}\right\}$ and let $W_{x}$ denote the subgroup generated by $\left\{s_{i} \mid i \in \sigma(x)\right\}$. For $x \in M-N$, let $\sigma(x)=\emptyset$ and let $W_{x}$ be the trivial subgroup. The space $\mathcal{U}(M, N, G)$ is defined by

$$
\mathcal{U}(M, N, G)=(W \times M) / \sim
$$

where $\sim$ is the equivalence relation defined by $(w, x) \sim\left(w^{\prime}, x^{\prime}\right)$ if and only if $x=x^{\prime}$ and $w^{-1} w^{\prime} \in W_{x}$. The image of $(w, x)$ in $\mathcal{U}(M, N, G)$ is denoted $[w, x]$. For $[w, x]$ in $\mathcal{U}(M, N, G)$ and $(v, g)$ in $W \rtimes G$, the action of $W \rtimes G$ on $\mathcal{U}(M, N, G)$ is defined by

$$
(v, g) \cdot[w, x]=[v w, g x] .
$$

(In the case where $G$ is the trivial group, a more general version of this construction had previously been described by Tits [18] and Vinberg [20].)

Some important features of this construction are:

- If $M$ is contractible, then so is $\mathcal{U}(M, N, G)$.

- If $M$ is an $n$-manifold with boundary and $\partial M=N$, then $\mathcal{U}(M, N, G)$ is an $n$-manifold.

- If $N$ is a finite complex and $M=\operatorname{Cone}(N)$, the cone on $N$, then $\mathcal{U}(\operatorname{Cone}(N), N, G)$ has a natural $C A T(0)$ cubical structure so that the link of each vertex is isomorphic to $N$ and so that $W \rtimes G$ is a group of isometries.

The proofs of these facts, in the case where $G$ is the trivial group, can be found in [8] and [11]. (See also [9], [10] and [12].)

Let us say that the $G$-action on $N$ is admissible if for every simplex $\sigma$ of $N$, the setwise and pointwise stabilizers of $\sigma$ are equal. (This can always be achieved by passing to the barycentric subdivision of $N$, cf., [3, p. 116].) Assume that the $G$-action on $N$ is admissible. For any subgroup $H$ of $G$, let $W^{H}$ denote the subgroup of $W$ generated by the vertices of $N$ which are fixed by $H$ (i.e., $W^{H}$ is the Coxeter group corresponding to the flag complex $\left.N^{H}\right)$. Then for any finite subgroup $H$ of $G$ we have the following:

- The fixed set of $H$ on $\mathcal{U}(M, N, G)$ is $\mathcal{U}\left(M^{H}, N^{H}, V_{H}\right)$, where, as before, $V_{H}=N_{G}(H) / H$.

- $W^{H} \rtimes V_{H}=N_{W \rtimes G}(H) / H$.

\section{The equivariant Bestvina-Brady CONSTRUCtion}

We recall the construction of [1] and its extension in [13]. This time, suppose we are given as data:

- A finite flag complex $L$.

- A group $Q$ of admissible symmetries of $L$. 
To this data we will associate a right-angled Artin group $A_{L}$ and a $C A T(0)$ space $X_{L}$ on which $A_{L} \rtimes Q$ is represented as a discrete, cocompact group of isometries (so $X_{L}=\underline{E} G$ where $G=A_{L} \rtimes Q$ ). We will also have a subgroup $B_{L}$ of $A_{L}$ and a subspace $Y_{L}$ of $X_{L}$ so that $B_{L}$ acts cocompactly on $Y_{L}$.

Let $I$ denote the vertex set of $L$. The group $A_{L}$ has generating set $\left\{a_{i}\right\}_{i \in I}$ and a relation of the form $\left[a_{i}, a_{j}\right]=1$, whenever $\{i, j\}$ spans an edge of $L$. Let $\phi: A_{L} \rightarrow \mathbb{Z}$ be the homomorphism which sends each generator $a_{i}$ to 1 . The kernel of $\phi$, denoted by $B_{L}$, is the Bestvina-Brady group associated to $L$. The $Q$-action on $I$ induces a $Q$-action on $A_{L}$ (through automorphisms) and the subgroup $B_{L}$ is $Q$-stable. Hence, we can form the semidirect products $A_{L} \rtimes Q$ and $B_{L} \rtimes Q$. The homomorphism $\phi$ extends to a homomorphism (also denoted by $\phi$ ) from $A_{L} \rtimes Q$ to $\mathbb{Z}$ by sending $Q$ to 0 . As in [6], $A_{L}$ is a cocompact group of isometries of a $C A T(0)$ cubical complex $X_{L}$. Since $A_{L}$ is torsion-free, $X_{L} / A_{L}=B A_{L}$. The $Q$-action on $L$ induces an isometric action on $X_{L} / A_{L}$ and it is easily checked that the group of all lifts of this action to $X_{L}$ is just $A_{L} \rtimes Q$. As in [1], there is a $\phi$-equivariant map $f: X_{L} \rightarrow \mathbb{R}$. In general, the homotopy type of the level set $f^{-1}(t)$ will vary as $t$ varies in $\mathbb{R}$; however, there is one important case when it does not: when $L$ is contractible. In any case, fix a $t \in \mathbb{R}$ and let $Y_{L}$ denote the level set $f^{-1}(t)$.

Assume that the $Q$-action on $L$ is admissible. As explained in [13], in the above construction, the process of taking fixed sets of $Q$ works as one might predict, i.e.,

$$
\begin{aligned}
\left(X_{L}\right)^{Q} & =X_{L^{Q}} \\
\left(Y_{L}\right)^{Q} & =Y_{L^{Q}}
\end{aligned}
$$

It was proved in [7] that the subgroup of $A_{L}$ fixed by $Q$ is $A_{L^{Q}}$ and then observed in [13] that it follows from this that the subgroup of $B_{L}$ fixed by $Q$ is $B_{L^{Q}}$. If we regard $Q$ as a subgroup of $B_{L} \rtimes Q$ and if $V_{Q}$ stands for $N_{B_{L} \rtimes Q}(Q) / Q$, then $V_{Q}=B_{L^{Q}}$ (cf., [13]).

The main result of [1] is that $B_{L}$ is type $\mathrm{F}$ if and only if $L$ is contractible. The main idea of [13] is that if $L$ is contractible but the $Q$-action is such that $L^{Q}$ is not contractible, then $B_{L} \rtimes Q$ will not be type $\mathcal{V} F$ (since $V_{Q}$ will not be type VF). In addition, it is proved in [13] that if $L^{Q}=\emptyset$, then $B_{L} \rtimes Q$ contains an infinite number of conjugacy classes of subgroups isomorphic to $Q$ (all of which are conjugate in $A_{L} \rtimes Q$ ). Furthermore, there are many examples of such $Q$-actions on contractible finite simplicial complexes $L$; for example, see [3] and [17]. We describe one of them below.

Example 1. Poincaré's homology 3-sphere, $M^{3}$, can be constructed by identifying opposite faces of a dodecahedron via a $\pi / 5$ rotation. Let $Q$ be the group of orientation preserving symmetries of the dodecahedron. ( $Q$ is isomorphic to $A_{5}$, the alternating group of degree 5.) It is well-known (e.g., see [3, pp. 55-58]) that $Q$ acts on $M^{3}$ with precisely one fixed point (the center of the dodecahedron). Hence, $Q$ acts on $L$, where $L$ is defined as the complement of the interior of the dodecahedron in $M^{3}$. The subcomplex $L$ is a 
2-dimensional cell complex with pentagonal 2-cells. Since $M^{3}$ is a homology sphere, $L$ is acyclic. Since we have deleted the fixed point, $L^{Q}=\emptyset$. Finally, $Q$-equivariantly triangulate $L$ as a flag complex (for example, by taking its barycentric subdivision). This gives an example to which we can apply the equivariant Bestvina-Brady construction as above.

\section{The First Class of EXAMPLes}

Let $Y_{L}$ be one of the examples of [13]. To simplify notation, set $\widetilde{Y}=Y_{L}$, $\Gamma=B_{L} \rtimes Q$ and $Y=Y_{L} / B_{L}=\widetilde{Y} / B_{L}$. Thus, $Y$ is a finite complex with fundamental group $B_{L}$ and with universal cover $\widetilde{Y}$; the finite group $Q$ acts cellularly on $Y$ and the group of all lifts of the $Q$-action to $\widetilde{Y}$ is $\Gamma$.

We want to $Q$-equivariantly "thicken" $Y$ to a compact manifold $M$ with boundary $N$. There are at least two methods for doing this. One is to use a theorem of Mostow [16] to $Q$-equivariantly embed $Y$ in a linear representation of $Q$ on some Euclidean space $\mathbb{R}^{n}$. (A proof of Mostow's Theorem can be found in [3, pp. 110-112].) Then choose a $Q$-equivariant triangulation of $\mathbb{R}^{n}$ and let $M$ be a regular neighborhood of $Y$ in $\mathbb{R}^{n}$. Another method is to give $Y$ the structure of a $Q$-CW complex and then, as in [17], build the thickening $M$ by using $Q$-handles attached by maps, which are $Q$-homotopic to the attaching maps for $Y$. In either case, we obtain a $Q$-action on $M$, a compact manifold with boundary, such that $M$ equivariantly deformation retracts onto $Y$. Equivariantly triangulate the boundary $N$ as a flag complex. Let $\widetilde{M}$ denote the universal cover of $M$ and let $\widetilde{N}$ denote the inverse image of $N$ in $\widetilde{M}$ (give $\widetilde{N}$ the induced triangulation). The group of all lifts of the $Q$-action to $\widetilde{M}$ is $\Gamma$ (since this is true for the $Q$-action on $Y$ and $Y$ is $Q$-homotopy equivalent to $M$.)

Now we apply the equivariant reflection group trick of Section 2. First consider the manifold $\mathcal{U}(M, N, Q)$. The group which acts on it is $W \rtimes Q$ where $W$ is the right-angled Coxeter group associated to $N$. The commutator subgroup $T$ of $W$ is a torsion-free subgroup of finite index. The manifold $\mathcal{U}(\widetilde{M}, \widetilde{N}, \Gamma)$ is clearly a covering space of $\mathcal{U}(M, N, Q)$. It is contractible since $\widetilde{M}$ is. The group which acts on $\mathcal{U}(\widetilde{M}, \widetilde{N}, \Gamma)$ is $\widetilde{W} \rtimes \Gamma$, where $\widetilde{W}$ is rightangled Coxeter group associated to $\widetilde{N}$. The quotient space is $M / Q$ which is compact. Let $\widetilde{T}$ denote the inverse image of $T$ in $\widetilde{W}$. It is a torsion-free subgroup of finite index in $\widetilde{W}$. So, $\widetilde{T} \rtimes B_{L}$ is a torsion-free subgroup of finite index in $\widetilde{W} \rtimes \Gamma$. Thus, $\widetilde{W} \rtimes \Gamma$ is type VF. This gives the following more precise version of Theorem 1 .

Theorem 3. The virtually torsion-free group $\widetilde{W} \rtimes \Gamma$ acts properly and cocompactly on the contractible manifold $\mathcal{U}(M, N, Q)$. Moreover,

(1) If $L^{Q}$ is empty, then $\widetilde{W} \rtimes \Gamma$ contains infinitely many conjugacy classes of finite subgroups isomorphic to $Q$. 
(2) If $L^{Q}$ is nonempty and not contractible, then $N_{\widetilde{W} \rtimes \Gamma}(Q) / Q$ contains a torsion-free subgroup of finite index which retracts onto $B_{L^{Q}}$ and hence is not type $F$

So, in either case, $\widetilde{W} \rtimes \Gamma$ is not type $\mathcal{V} F$.

Proof. If $L^{Q}=\emptyset$, then $\Gamma$ contains infinitely conjugacy classes of subgroups isomorphic to $Q$ and therefore, the same is true for the semidirect product $\widetilde{W} \rtimes \Gamma$. As explained in Section 2,

$$
N_{\widetilde{W} \rtimes \Gamma}(Q) / Q=\widetilde{W}^{Q} \rtimes V_{Q}
$$

and as explained in Section $3, V_{Q}=B_{L^{Q}}$. Hence, for a suitable torsion-free subgroup $\widetilde{T}^{Q}$ of finite index in $\widetilde{W}^{Q}$, the subgroup $\widetilde{T}^{Q} \rtimes B_{L^{Q}}$ retracts onto $B_{L^{Q}}$.

\section{The SeCOND Class of EXAMPles}

In this section, the finite group will act on a sphere rather than on a contractible complex. Again we use the equivariant reflection group trick of Section 2. Suppose that $N$ is a triangulation of the $(n-1)$-sphere as a flag complex and that a finite group $G$ acts admissibly, by simplicial automorphisms on $N$. So, Cone $(N)$ will be an $n$-disk. To simplify notation, set $\Sigma=\mathcal{U}(\operatorname{Cone}(N), N, G)$. Then $\Sigma$ is an $n$-dimensional $C A T(0)$ manifold equipped with an isometric action of $W \rtimes G$. As we noted in the Introduction, this implies that $W \rtimes G$ is type $\mathcal{V} F$.

For any finite subgroup $H$ of $G$ we have

$$
\Sigma^{H}=\mathcal{U}\left(\operatorname{Cone}\left(N^{H}\right), N^{H}, V_{H}\right)
$$

where $V_{H}=N_{G}(H) / H$. One of the main results of [9] is a calculation of the cohomology with compact supports of such spaces. In particular, Theorem A of [9] yields

$$
H_{c}^{i}\left(\Sigma^{H}\right)=\sum_{w \in W^{H}} \bar{H}^{i-1}\left(N^{H}-\sigma(w)\right)
$$

where $\sigma(w)$ is a certain simplex of $N^{H}$ depending on $w \in W^{H}$. It follows from this formula (cf., Theorem B in [9]) that the subgroup $H$ of $W \rtimes G$ satisfies the condition of the definition in Section 1 if and only if $N^{H}$ is a "generalized homology sphere", in that sense that it is a homology manifold with the same homology as a sphere. So, we have proved the following.

Theorem 4. The group $W \rtimes G$ is a virtual Poincaré duality group. It is a $\mathcal{V} P D$-group if and only if $N^{H}$ is a generalized homology sphere for each finite subgroup $H$ of $G$.

Since there are many examples of finite group actions on spheres where the fixed sets do not have the homology of a sphere (see [3]), Theorem 4 implies Theorem 2. We give a concrete example below. 
Example 2. Let $N\left(a_{1}, \ldots, a_{n}\right)$ denote the link of the origin in the complex hypersurface

$$
\left(z_{1}\right)^{a_{1}}+\cdots+\left(z_{n}\right)^{a_{n}}=0 .
$$

It is a smooth manifold of dimension $2 n-3$, called a "Brieskorn manifold". As Brieskorn showed, it is often homeomorphic to $S^{2 n-3}$, see [15]. For example, $N=N(3,2,2,2)$ is diffeomorphic to $S^{5}$. If $\zeta$ denotes a sixth root of unity, then define an action of $\mathbb{Z} / 6$ on $N$ by the formula

$$
\zeta \cdot\left(z_{1}, z_{2}, z_{3}, z_{4}\right)=\left(\zeta^{2} z_{1}, \zeta^{3} z_{2}, z_{3}, z_{4}\right) .
$$

The fixed set of $\mathbb{Z} / 3$ on $N$ is $N(2,2,2)$ which is $\mathbb{R} P^{3}$, the fixed set of $\mathbb{Z} / 2$ is $N(3,2,2)$ which is a 3 -dimensional lens space with fundamental group $\mathbb{Z} / 3$ and the fixed set of $\mathbb{Z} / 6$ is $N(2,2)$ which is the disjoint union of two circles. Equivariantly triangulate $N$ as a flag complex and form the $C A T(0)$ 6-manifold $\Sigma$ as above. For any subgroup $H$ of $\mathbb{Z} / 6$, let $\widetilde{V}_{H}=W^{H} \rtimes V_{H}$ be the group corresponding to $H$. For $H=\mathbb{Z} / 3$ or $\mathbb{Z} / 2, \widetilde{V}_{H}$ has $c d_{\mathbb{Q}}=4$ and $H^{4}\left(\widetilde{V}_{H} ; \mathbb{Z} \widetilde{V}_{H}\right)=\mathbb{Z}$. However, $H^{3}\left(\widetilde{V}_{H} ; \mathbb{Z} \widetilde{V}_{H}\right)$ is either an infinite sum of $\mathbb{Z} / 2$ 's (when $H=\mathbb{Z} / 3$ ) or of $\mathbb{Z} / 3$ 's (when $H=\mathbb{Z} / 2$ ). For $H=\mathbb{Z} / 6$, the group $\widetilde{V}_{H}$ is of cohomological dimension 2 and its second cohomology group is free abelian of infinite rank. So, $W \rtimes G$ is not a $\mathcal{V P D}$-group.

\section{APPENDIX: TYPE $\mathcal{V} F P$}

The orbit category, $\mathcal{O}(G)$, of a discrete group $G$ is the category whose objects are the $G$-sets $G / H$ for $H$ any subgroup of $G$, with morphisms the $G$-maps between these $G$-sets. This is equivalent to a category whose objects are all transitive $G$-sets. The proper orbit category, $\mathcal{O}$ or $\mathcal{O}(G, \mathcal{F})$, is the full subcategory of $\mathcal{O}(G)$ whose objects are the transitive $G$-sets $G / H$ for $H$ finite. This is equivalent to a category whose objects are all transitive proper $G$-sets. A Bredon coefficient system for proper $G$-spaces or a $\mathbb{Z O}$ module is a contravariant functor from $\mathcal{O}$ to abelian groups, and a morphism of $\mathbb{Z O}$-modules is a natural transformation between two such functors. The category of $\mathbb{Z O}$-modules is an abelian category.

The trivial $\mathbb{Z O}$-module $\mathbf{Z}$ is defined to be the constant functor on $\mathcal{O}$ that sends every object to $\mathbb{Z}$ and every morphism to the identity map of $\mathbb{Z}$. For each object $G / H$ of $\mathcal{O}$, define a $\mathbb{Z O}$-module $P_{G / H}$ by

$$
P_{G / H}(-)=\mathbb{Z M a p}_{G}(-, G / H),
$$

so that the value of $P_{G / H}$ on $G / K$ is the free abelian group with basis the $G$-maps from $G / K$ to $G / H$. It may be shown that each $P_{G / H}$ is projective, and that any $\mathbb{Z} \mathcal{O}$-module is the homomorphic image of some direct sum of these modules. Call $P_{G / H}$ the free $\mathbb{Z} \mathcal{O}$-module of rank one based at $G / H$, and define a finitely generated free $\mathbb{Z} \mathcal{O}$-module to be a finite direct sum of modules $P_{G / H}$. A finitely generated projective $\mathbb{Z} \mathcal{O}$-module is a direct summand of a finitely generated free $\mathbb{Z} \mathcal{O}$-module. 
Definition. A group $G$ is type $\mathcal{V} F P$ (resp. type $\mathcal{V} F L$ ) if the trivial $\mathbb{Z} \mathcal{O}$ module $\mathbf{Z}$ admits a finite resolution by finitely generated projective $\mathbb{Z O}$ modules (resp. finitely generated free $\mathbb{Z} \mathcal{O}$-modules).

For a $G$-space $X$, the $H$-fixed points $X^{H}$ may be identified with the space $\operatorname{Map}_{G}(G / H, X)$, and the map

$$
G / H \mapsto \operatorname{Map}_{G}(G / H, X)
$$

describes a contravariant functor from $\mathcal{O}$ to spaces. For a $G \mathrm{CW}$-complex, the map

$$
G / H \mapsto C_{*}\left(\operatorname{Map}_{G}(G / H, X)\right)
$$

describes a chain complex of free $\mathbb{Z} \mathcal{O}$-modules, which are finitely generated if $X$ contains finitely many orbits of cells. This construction yields a free resolution of $\mathbf{Z}$ when applied to $\underline{E} G$, and hence any group of type $\mathcal{V} F$ is of type $\mathcal{V} F L$.

For more information concerning the category of $\mathbb{Z} \mathcal{O}$-modules, see [14], which contains proofs of all the statements made in this section.

\section{REFERENCES}

[1] M. Bestvina and N. Brady, Morse theory and finiteness properties of groups, Invent. Math. 129 (1997), 445-470.

[2] N. Brady, I. J. Leary and B. E. A. Nucinkis, On algebraic and geometric dimension for groups with torsion, J. London Math. Soc. 64 (2001), 489-500.

[3] G. Bredon, Introduction to Compact Transformation Groups, Academic Press, New York and London, 1972.

[4] M. Bridson and A. Haefliger, Metric Spaces of Nonpositive Curvature, SpringerVerlag, Berlin, Heidelberg and New York, 1999.

[5] K.S. Brown, Cohomology of Groups, Graduate Texts in Math. 87, Springer-Verlag, Berlin, Heidelberg and New York, 1982.

[6] R. Charney and M.W. Davis, Finite $K(\pi, 1)$ s for Artin groups, Prospects in Topology (ed. F. Quinn), Annals of Math. Studies 138, Princeton Univ. Press, Princeton, 1995, pp. 597-627

[7] J.S. Crisp, Symmetrical subgroups of Artin groups, Adv. Math. 152 (2000), 159-177.

[8] M.W. Davis, Groups generated by reflections and aspherical manifolds not covered by Euclidean space, Ann. of Math. (2) 117(1983), 293-325.

[9] M.W. Davis, The cohomology of a Coxeter group with group ring coefficients, Duke Math. J. 91 (1998), 297-314.

[10] M.W. Davis, Poincaré duality groups, Surveys in Surgery Theory, Vol. 1 (eds. S. Cappell, A. Ranicki, J. Rosenberg), Ann. of Math. Studies 145, Princeton Univ. Press, Princeton, 2000, pp. 167-193.

[11] M.W. Davis and G. Moussong, Notes on nonpositively curved polyhedra, Low Dimensional Topology (eds. K. Boroczsky, W. Neumann, A. Stipicz), Bolyai Soc. Math. Studies 8, Budapest, 1999, pp. 11-94.

[12] M. Gromov, Hyperbolic groups, Essays in Group Theory (ed. S.M. Gersten) M.S.R.I. Publ. 8 Springer-Verlag, Berlin, Heidelberg and New York, 1987.

[13] I. J. Leary and B. E. A. Nucinkis, Some groups of type VF, to appear in Invent. Math.

[14] W. Lück, Transformation groups and algebraic K-theory, Lecture Notes in Mathematics 1408, Springer-Verlag, Berlin Heidelberg and New York, 1989. 
[15] J. Milnor, Singular Points of Complex Hypersurfaces, Ann. of Math. Studies 61, Princeton Univ. Press, Princeton, 1968.

[16] G. Mostow, Equivariant embeddings in euclidean space, Ann. of Math. 65 (1957), 432-446.

[17] R. Oliver, Fixed-point sets of group actions on finite acyclic complexes, Comment. Math. Helv. 50 (1975), 155-177.

[18] J. Tits, On buildings and their applications, Procceedings of the ICM, Vancouver 1974, vol. 1, Canad. Math. Congress, Montreal, 1975, pp. 209-220.

[19] T. tom Dieck, Orbittypen und äquivariante Homologie I, Arch. Math. 23 (1972), 307-317.

[20] E.B. Vinberg, Discrete linear groups generated by reflections, Math. USSR Izvestija 5 No. 5 (1971), 1083-1119.

Department of Mathematics, The Ohio State University, $231 \mathrm{~W} .18 \mathrm{Th}$ Avenue, Columbus, Ohio 43210, United States

E-mail address: mdavis@math.ohio-state.edu

Faculty of Mathematical Studies, University of Southampton, SouthampTON, SO17 1BJ, United KINGDOM

E-mail address: I.J.Leary@maths.soton.ac.uk 